\title{
Accuracy of a Flying Cutting Device
}

\section{Christian Munzinger ${ }^{1, a}$, Juergen Fleischer ${ }^{1, b}$, Gregor Stengel $^{1, \mathrm{c}}$, Markus Schneider ${ }^{1, d}$}

${ }^{1}$ wbk Institute of Production Science, Universität Karlsruhe (TH), Kaiserstr. 12, D-76131 Karlsruhe, Germany

amunzinger@wbk.uka.de, ${ }^{b}$ fleischer@wbk.uka.de, ${ }^{c}$ stengel@wbk.uka.de, ${ }^{d}$ schneider@wbk.uka.de

Keywords: Control, Industrial Robots, Accuracy, Calibration

\begin{abstract}
The prototype for the flying cutting of spatially curved extrusion profiles developed as part of the Collaborative Research Center Transregio 10 (SFB/TR 10) was tested as an integrated part of the overall system in first test runs. The profiles resulting from this process give proof of the potential involved in both, the novel curved profile extrusion (CPE) and the automatic supporting and cutting device. For subsequent automated processing to become possible, however, the reliably achievable accuracy of extruded profiles needs to be further improved. By the example of the extruded profiles produced so far, this article discusses potential factors that may impair profile accuracy and presents approaches and methods for the improvement of accuracy.
\end{abstract}

\section{Introduction}

The previous grant period of the Collaborative Research Center Transregio 10 (SFB/TR 10) was dedicated to a study of the theoretical basics and the elaboration of concepts for a process chain for the flexible production of three-dimensionally curved aluminium profiles.

During the first phase of the Collaborative Research Center Transregio 10, subproject A4 conceived and implemented a prototype for a cutting and guiding device required for the novel "multiaxis CPE" [1]. The prototype of the system includes a so-called flying saw and a transfer tool, which are used as tools for a standard industrial robot (Figure 1). The prototype of the flying cutting device allows for the guiding and cutting of three-dimensionally curved profiles [2]. The production of spatially curved profiles requires the relevant forces of gravity and acceleration to be absorbed by the flying cutting device in order to prevent undesired deformation that might otherwise be caused by these forces. Neither may there be any influences resulting from the cutting process that affect the profile during manufacturing. Besides, accelerations and velocities higher than in mere robot applications can be achieved through the use of highly dynamic additional axes in the tool of the flying saw und the transfer tool. The transfer tool allows the semi-automatic transfer of the cut profiles to other machining points [3]. 


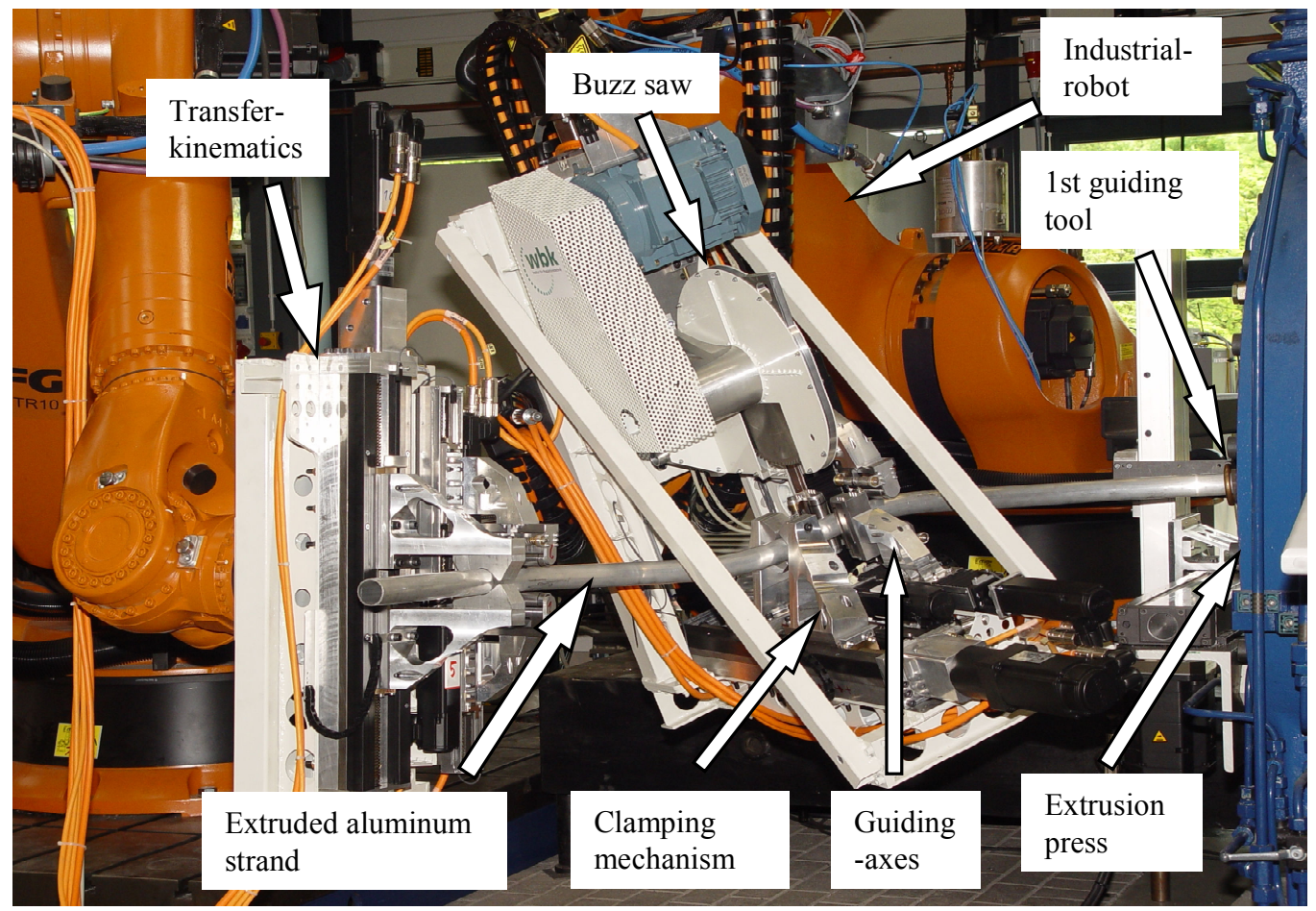

Figure 1: Prototype of the flying cutting device (wbk, iul) [2]

All kinematic mechanisms involved in the production process need to be operated synchronously in terms of their movements in order to guarantee the reactionless support of the profile during the actual production process. In case of the prototypically implemented system presented here, this is ensured through a primary NC control. The NC control takes care of the synchronous activation of all kinematic mechanisms involved in the process on the basis of its design as an electronic transmission. During this process, the NC control transfers the reference paths identified in an offline programming environment to the respective axes in real time [4].

In the summer of 2006, the overall system comprising both the extrusion press and the guiding tool belonging to subproject A1, "multi-axis CPE", which is located in Dortmund, and the flying cutting device and kinematic transfer mechanism from subproject A4, located in Karlsruhe, was set up in a process chain for the first time. In first tests, the functionability of this concept regarding the production of aluminium extruded profiles was proved.

\section{Motivation and Objective}

The initial focus during the first grant period was the verification of the feasibility of the fly-cutting process. Towards the end of the first grant period, first trials with the overall system produced extruded profiles which were measured in order to determine the profile contour accuracy achieved so far. The measurements were performed with a coordinate measurement arm. The identified actual profile contour data was then compared with the reference contour from the CAD. The deviation of the measured profiles as compared to the reference geometry was measured at 14 measuring points for the purpose of evaluation. Figure 2 - top shows an example of a measured profile in comparison with the reference contour. A summary of the other results can be seen from Figure 2 - bottom. 


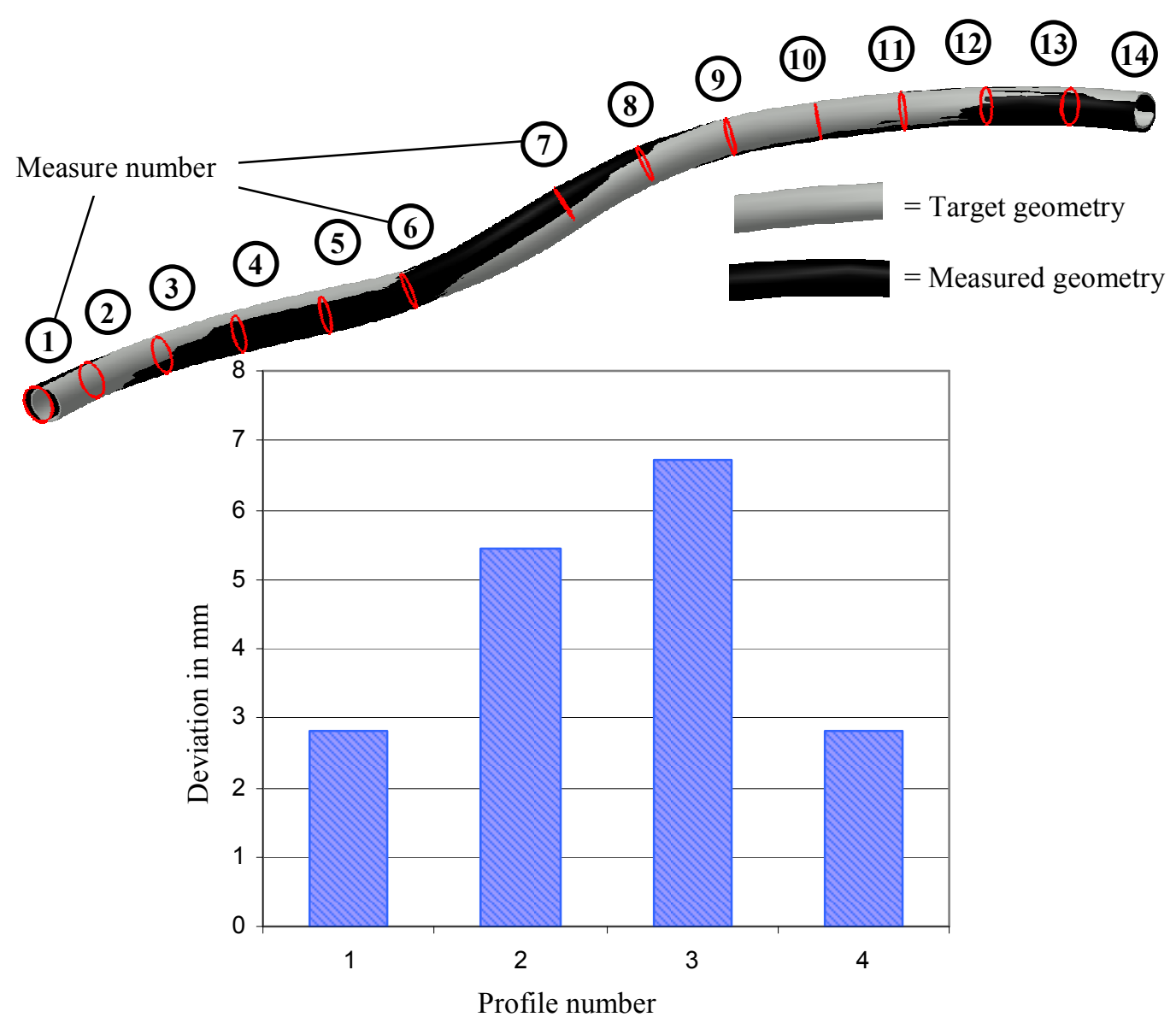

Figure 2: Measured profile contour (above, wbk), summary of measurements (below, wbk)

The deviations shown by the extruded profiles resulting from the process chain of the subprojects A1 and A4 combined are similar to the contour deviations of the extruded profiles that have so far been produced by subproject A1 with manual support of the profiles [5]. The medium error in terms of contour deviation across all measured profiles amounted to a deviation of $4.5 \mathrm{~mm}$ for a profile length of approx. $1400 \mathrm{~mm}$ and a radius of $560 \mathrm{~mm}$.

The current funding period is aimed at increasing the accuracy of the profile contour, because the accuracies achieved with the flying cutting device so far are insufficient for subsequent automated machining processes. For this goal to be achieved, the first step to be taken is a detailed analysis of the entire process. To that end, preliminary investigations are required to identify the boundary conditions during the operation of the flying cutting device and to determine the main influencing factors affecting the accuracy of the profile contour. Based on the results of the analysis, concepts for the calibration of the flying cutting device and the kinematic transfer mechanism are then conceived and implemented.

\section{Influencing factors}

Studies of the overall system facilitate the identification of a high number of factors impairing accuracy that result from various system components. Some of these factors regarding CPE have already been presented in [6] and [7]. The effects of gravity and profile cantilever length on profile contour deviation were studied. Besides, a first estimation regarding the possible effects of tilting the first guiding tool was carried out. There are additional influencing factors for the overall process and the flying cutting device that can be seen from the following fault/effects diagram (see Figure 3). 


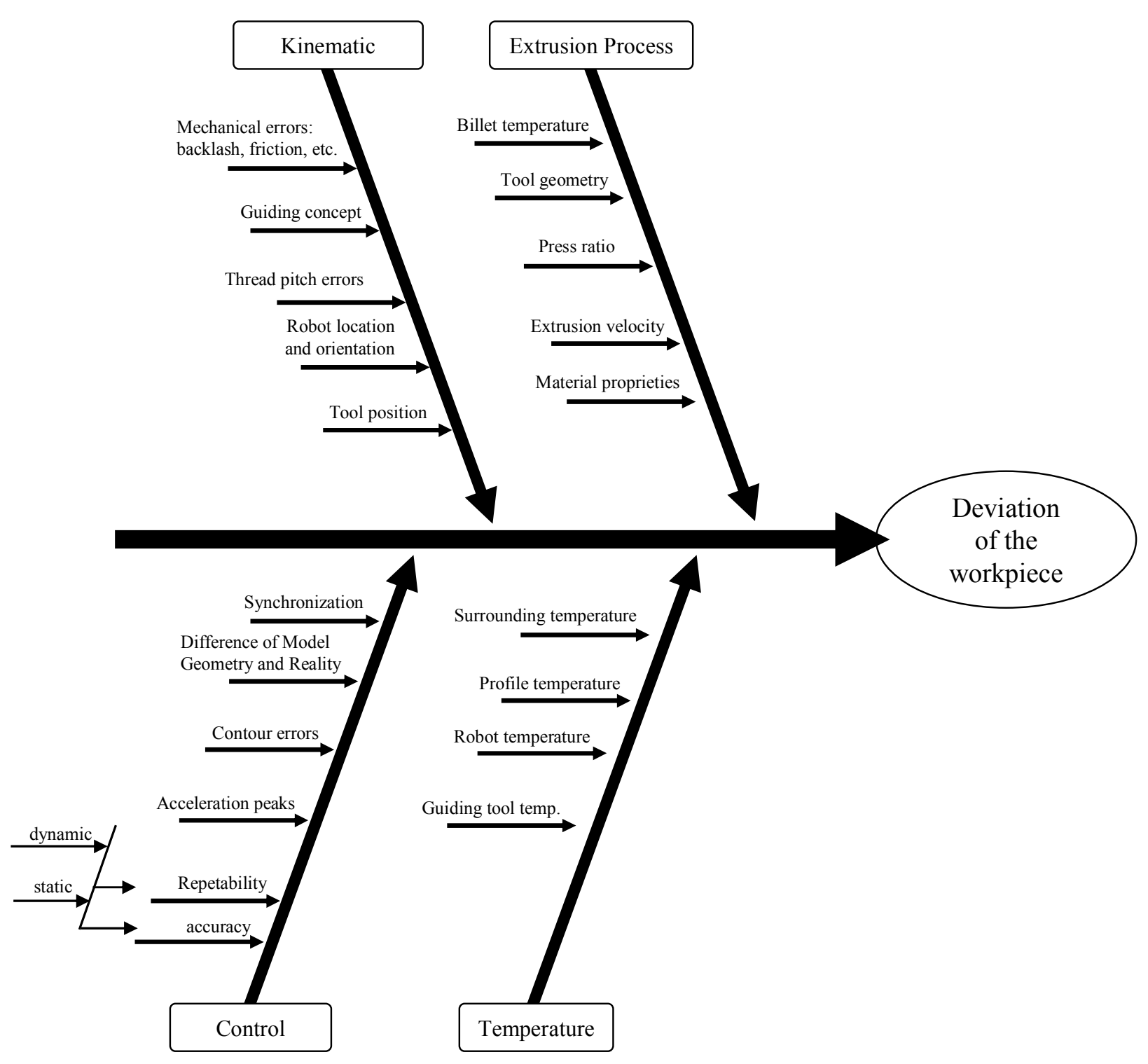

Figure 3: Fault/Effects diagram (wbk, following [6], [7], [8])

The factors affecting contour accuracy can be grouped in four error categories: kinematic mechanism, extrusion process, control and temperature. The enumeration of these influencing factors does not imply a rating in terms of either significance or quantitative effect of the contour deviation in case of occurrence of the respective error. The next working steps, therefore, consist in the identification and evaluation of the individual influencing factors. Subsequently, suitable methods for error compensation are to be elaborated and implemented.

Influences resulting from the kinematic mechanism such as an inclination of the robots or the guiding tool can be identified by adequate measuring methods. These usually repeated errors can then be minimized by an exact positioning of the components or by adjusting the control model.

The trials and operations presented in this article relate to the identification of the statistic and temperature-induced deviations of the employed kinematic mechanisms. Following projects shall deal with other influencing factors such as control and thermal workpiece deformation. 


\section{Static deviations of the kinematic mechanism}

For the identification of deviations at the TCP (Tool Center Point) of the flying cutting device, a laser tracker was used to record a point grid with a spacing of $100 \mathrm{~mm}$ between each point and a measuring envelope of $\mathrm{LxWxH}=1000 \mathrm{~mm} \times 1000 \mathrm{~mm} \times 800 \mathrm{~mm}$ in front of the extrusion press. For the calibration of the measuring envelope, the zero level and the corresponding Cartesian coordinate system were determined on the basis of three calibrated points. Subsequent measurements were carried out in the envelope that had thus been calibrated. The deviations identified for the measuring envelope can be seen in Figure 4.

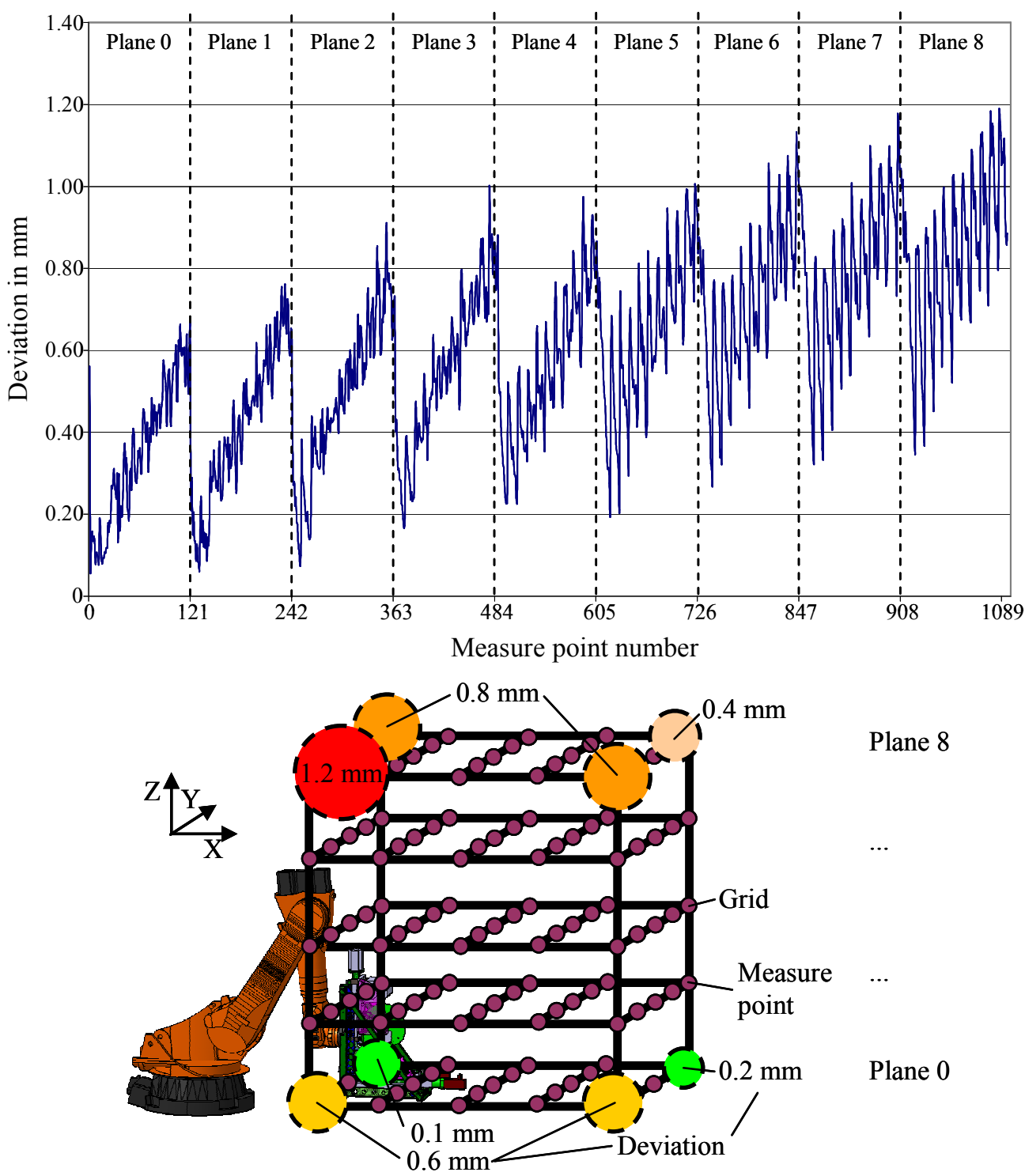

Figure 4: Identified absolute deviations in a measuring envelope of $1000 \mathrm{~mm} \times 1000 \mathrm{~mm} \times 800 \mathrm{~mm}$ (wbk)

The distribution of individual errors shows growing position deviations with increasing distance in $\mathrm{x}, \mathrm{y}$ and $\mathrm{z}$ directions. The absolute deviations from the actual positions amount to $1.2 \mathrm{~mm}$ in the upper section of the measured space (see marking Figure 4, bottom) and to up to approx. $0.6 \mathrm{~mm}$ in the lower section. The absolute deviation is calculated on the basis of the respective reference and actual components of the measurement values according to the following formula: 


$$
\text { Error_Absolute }=\sqrt{\left(x_{\text {nom }}-x_{\text {act }}\right)^{2}+\left(y_{\text {nom }}-y_{\text {act }}\right)^{2}+\left(z_{\text {nom }}-z_{\text {act }}\right)^{2}}
$$

Formula 1: Absolute error

The measurements substantiated a significant deviation of the actual position of the TCP of the flying cutting device regarding the absolute position. A compensation of these errors is indispensable if high accuracies of the profile contour should be achieved.

\section{An approach to the compensation of static deviations}

[9] and [10] present different methods for the achievement of industrial robot accuracy increases through calibration. Basically, these methods can be subdivided into numerical or data-based calibration and model-based static and dynamic calibration. A model-based static calibration is already performed by the robot manufacturer. One approach to a further increase of profile accuracy is, therefore, data-based or numerical calibration of the control and the overall system on the basis of correction values for path generation. Improving the system's accuracy by this method, the threedimensional working envelope is first subdivided into a grid structure. Afterwards, the respective position offset between reference and actual position can be determined for each grid position. By interpolating between the grid points the intermediate errors can be described, too. This method is mainly used for the calibration of machine tools because of the cartesian arrangement of axes which impedes any alteration of main axis orientation. With regard to the flying cutting device, the working envelope in front of the extrusion press can be assumed to be very limited because of the restrictions resulting from the CPE. The possible axis positions of the industrial robots are also limited by $\mathrm{CPE}$, which allows good results through numerical calibration according to [11], too.

The identified measurement values may now be used as a database for the creation of an error model. This error model can then be used for a numerical calibration of the motion paths of the flying cutting device. To that end, for the correction of the paths generated offline in accordance with the deviations identified with the error model, an additional process can be added after the path generation for the fly-cutting. The updated reference lists are then used by the primary NC control for control of the system.

\section{Influences resulting from a temperature increase caused by the extrusion press}

Temperature influences on the industrial robots and the guiding tools represent another cause of contour deviations on the profile accuracy. The extrusion process is based on an aluminum forming process at high temperatures of $450{ }^{\circ} \mathrm{C}$. Due to their position right in front of the extrusion press, the industrial robots and guiding tools used operate in an inhomogeneous temperature field and are heated from one side. Besides the intrinsic heating of the industrial robot in operation, the resulting deformation represents another source of errors regarding path deviations. One specific particularity of the CPE is significant when it comes to analyzing the thermal deviation behavior. Although the required robot path depends on the intended workpiece geometry, the probability of the robot being in front of the extrusion press is very high. In this section, any position deviations of the industrial robot have got a particularly high impact on the contour accuracy of the profiles because of the leverage effect in that area.

In order to reduce deviations in this working envelope, a robot position suitable with regard to thermal aspects was chosen in a first step. It is important to keep the distance between the flying saw and the robot base as small as possible. Furthermore, the height of the cutting device is supposed to match the height of the robot base as much as possible to allow the thermal expansions of both oscillations in $\mathrm{z}$ direction to compensate each other to the largest possible extent.

In order to examine the influences of this process-induced heating on the industrial robots in a second step, the robot cell for fly-cutting was additionally equipped with a heating zone used to simulate the temperature generated by an extrusion process in the industrial environment. The heat- 
ing zone consists of several ceramic heating elements connected in series which are continuously adjustable to temperatures from $20^{\circ} \mathrm{C}$ to $750{ }^{\circ} \mathrm{C}$. The heated section of the heating zone is approx. $1100 \mathrm{~mm} \times 900 \mathrm{~mm}$ large, and, thus, corresponds to the area used for the extrusion press of the Collaborative Research Center.

The pilot testing performed as part of subproject A1 identified the following temperatures at the actual extrusion press during operation:

\begin{tabular}{|l|l|}
\hline Measuring Position & Temperature \\
\hline Surface Temperature of Press & $80^{\circ} \mathrm{C}$ \\
\hline First Guiding Tool & $45,3^{\circ} \mathrm{C}$ \\
\hline $500 \mathrm{~mm}$ behind First Guiding Tool (Air Temp.) & $30,7^{\circ} \mathrm{C}$ \\
\hline $1000 \mathrm{~mm}$ behind First Guiding Tool (Air Temp.) & $28,3^{\circ} \mathrm{C}$ \\
\hline $1500 \mathrm{~mm}$ behind First Guiding Tool (Air Temp.) & $26,6^{\circ} \mathrm{C}$ \\
\hline Room Temperature & $24,4^{\circ} \mathrm{C}$ \\
\hline
\end{tabular}

Figure 5: Temperatures in the periphery of the extrusion press (iul/wbk)

According to these results, the air temperature in the periphery of the robot, which is situated about $800 \mathrm{~mm}$ away from the extrusion press, exceeds the regular room temperature by approximately $10 \mathrm{~K}$. In order to simulate the heat generated by the extrusion press, the heating zone was set to $100{ }^{\circ} \mathrm{C}$ surface temperature. At a distance of $450 \mathrm{~mm}$ from the heating zone, the temperature increase amounted to approx. $7 \mathrm{~K}$ at a level of $400 \mathrm{~mm}$ above the floor, whereas $1350 \mathrm{~mm}$ above the floor it was $13 \mathrm{~K} .1500 \mathrm{~mm}$ away from the heating zone, the temperature increase was still approx. $2 \mathrm{~K}$ at a level of $400 \mathrm{~mm}$ above the floor.

The industrial robot and the flying cutting device were then equipped with 45 temperature sensors to measure the surface temperature of the components. A 60-channel temperature measurement module designed for relative measurements was used for temperature sensing, allowing a 6 -second cycle of measuring-data acquisition with a resolution of $1 / 10^{\circ} \mathrm{C}$.

In order to measure TCP displacement and an alteration of the absolute and repetitive accuracies during operation of the flying cutting device with partial temperature impact, a point grid with a spacing of $400 \mathrm{~mm}$ between measuring points was approached periodically with the industrial robot in continuous operation within a working envelope of $\mathrm{LxWxH}=800 \mathrm{~mm} \times 800 \mathrm{~mm} \times 800 \mathrm{~mm}$ in front of the heating zone. The $\mathrm{x}, \mathrm{y}$ and $\mathrm{z}$ position of the TCP were identified by means of a laser tracker by the Faro company. The positions approached with the robot correspond to a working envelope in front of the extrusion press that is particularly important for compensation. Simultaneously, the temperature changes at the measuring points were recorded. Figure 6 shows the setup of the test rig. 


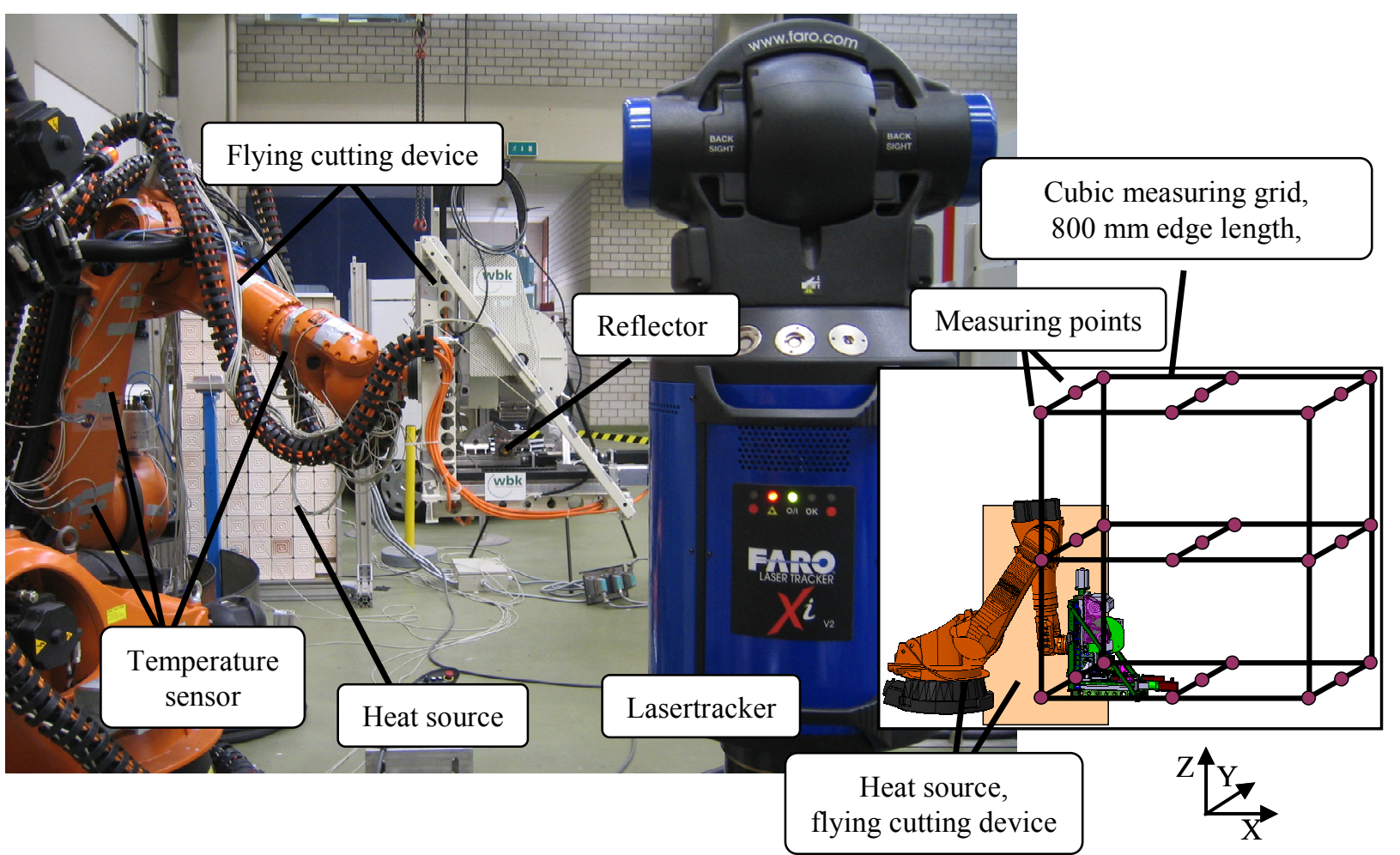

Figure 6: Test rig for the simulation of the temperature impact (wbk, left), schematic illustration of the measured points (wbk, right)

The measurements were discontinued once stable temperature conditions had been achieved, i.e. after approx. 6 hours. The results showed a maximum temperature increase of approx. $9 \mathrm{~K}$ at the robot and the flying cutting device in the upper section of the tool. Temperatures were generally higher in the front section of the system, which can be explained by the affected parts being exposed to direct radiation emanating from the heating zone. Figure 7 provides a summary of the results of the temperature increase at the side facing the heat source (front side).

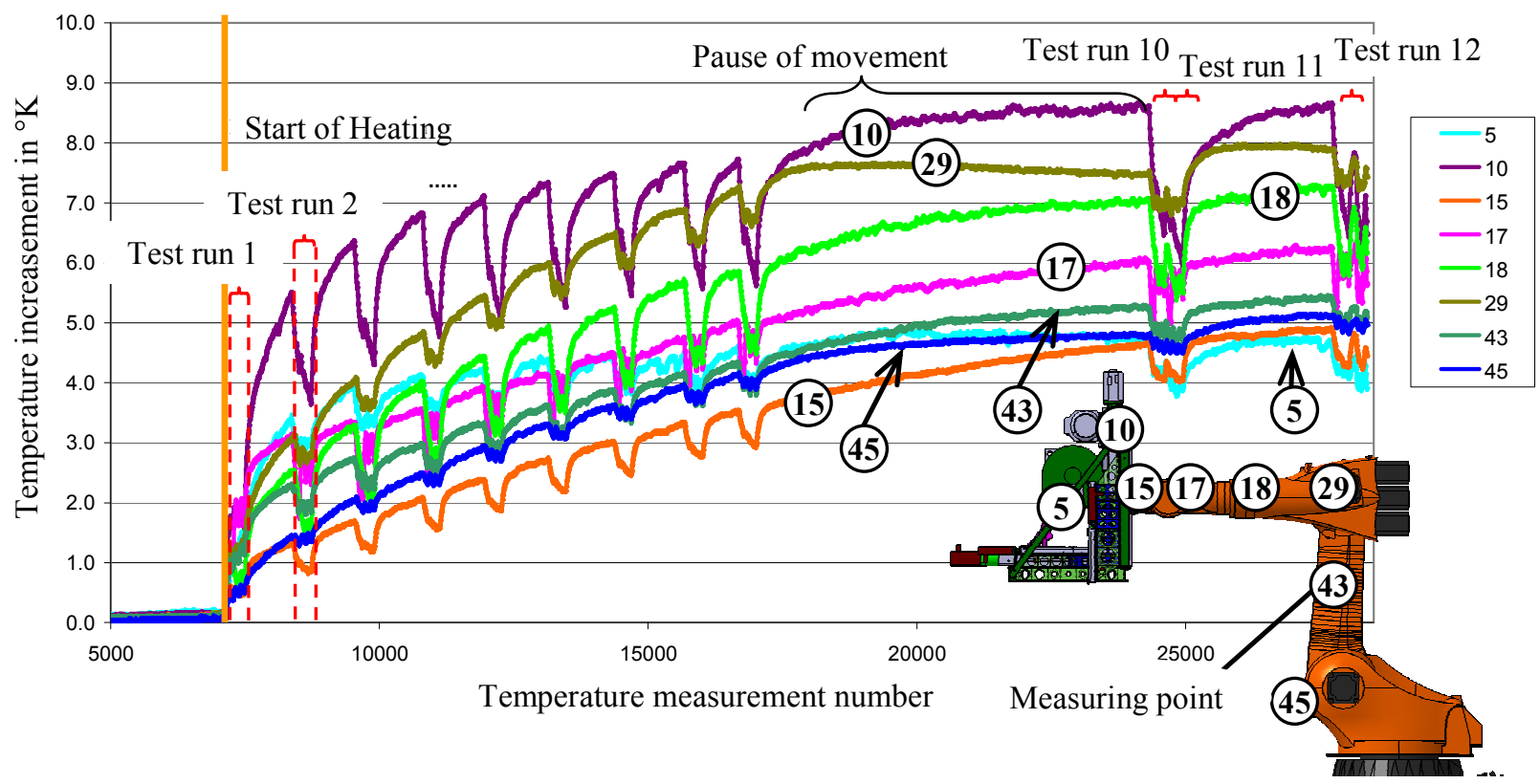

Figure 7: Temperature measurements, partial heating, front side (wbk)

The sawtooth-shaped temperature changes displayed in Figure 7 result from the measurement motion of the robot when recording the grid points. During the 5-minute long measurement motions, temperatures decrease by a maximum of $2 \mathrm{~K}$ each, whereas the temperature continues to in- 
crease during the 15 minute break between measurements while the robot is positioned in the starting spot in front of the heat source. Between measurements 9 and 10 there was a 45 minute break, and there was another 30 minute break between measurements 11 and 12 .

The temperature development along the upper robot arm includes measuring points 15, 17, 18 and 29 , the maximum temperature increase being $8 \mathrm{~K}$. Along the lower arm including measuring points 43 and 45, temperatures exceed the initial value by up to $5.2 \mathrm{~K}$. The temperature at the saw frame amounts to approx. $8.8 \mathrm{~K}$ at measuring point 10 and approx. $4.5 \mathrm{~K}$ at measuring point 5 in the steady-state condition. Figure 8 shows the temperatures on the back side facing away from the heating zone by way of comparison.

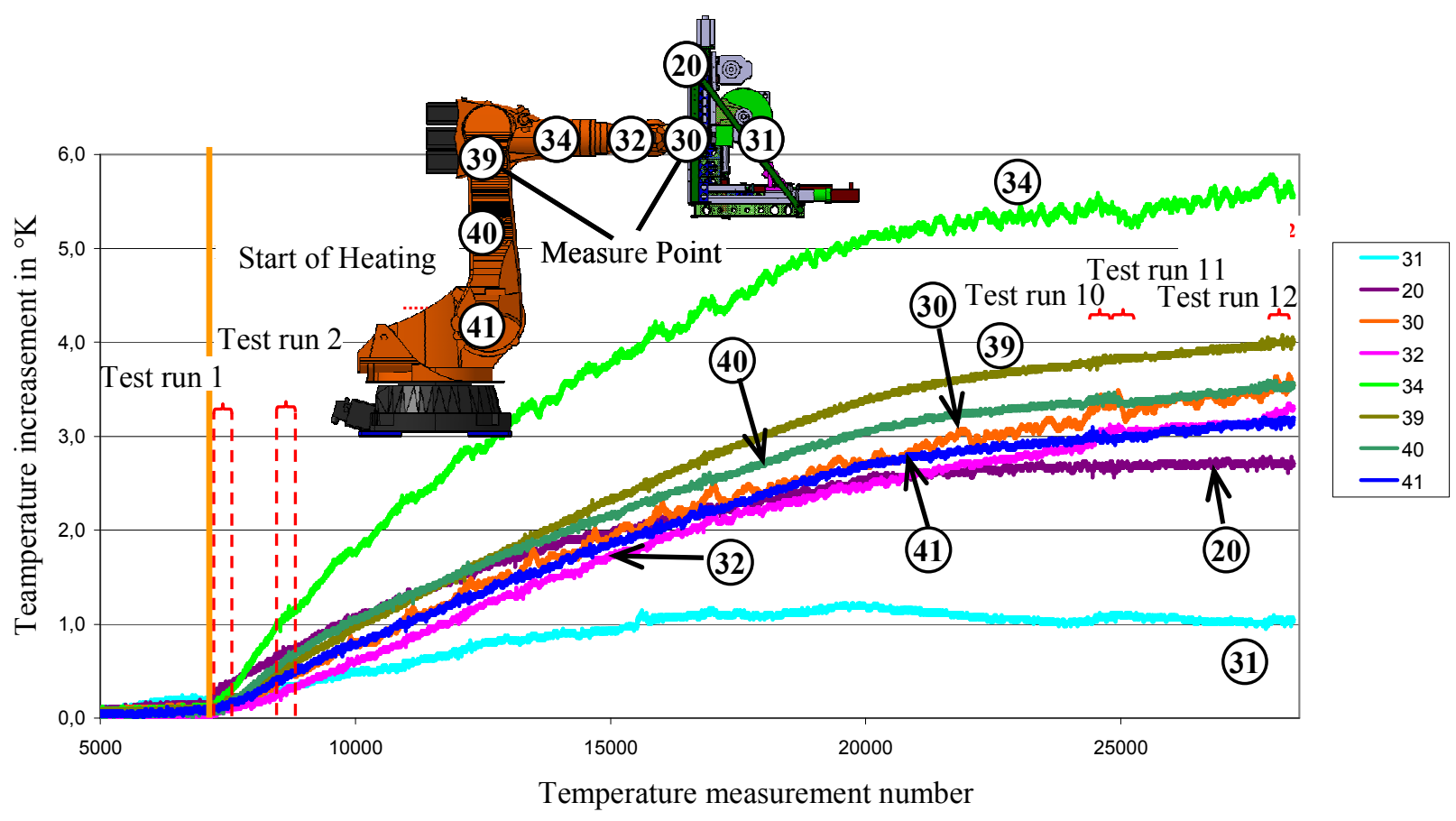

Figure 8: Temperature measurements, partial heating, back side (wbk)

The component surface temperature was generally lower on the back side than on the front side. There was no drop of temperatures during measurement motions like the decrease identified on the front side. Temperature distribution along the upper robot arm, beginning at the flange, exceeded the initial temperature by up to $5.8 \mathrm{~K}$ at the end of the measurements. Along the lower robot arm, the temperature increase amounted to a maximum of $4.0 \mathrm{~K}$ as compared to the initial temperature. The temperature at the saw frame had increased by approx. $2.8 \mathrm{~K}$ at measuring point 20 and by approx. $1 \mathrm{~K}$ at measuring point 31 at the end of the measuring process.

The measured temperature difference between front and back side of the robot amounted up to $4 \mathrm{~K}$ between measuring points 29 and 39. The maximum difference between front and back side at the fifth axis of the robot was approx. $2.9 \mathrm{~K}$ right at the upper robot arm. The difference at the remaining measuring points was between $1.3 \mathrm{~K}$ and $1.8 \mathrm{~K}$. The temperature difference between measuring point 5 on the front side and measuring point 31 on the back side was approx. $4 \mathrm{~K}$ at the longitudinal struts of the saw frame.

The heating caused by the simulated extrusion press led to non-uniform temperature increases across all robot axes. The temperature differences on the front and back side of the kinematic structure cause a change in length and a deformation of the individual axes through thermal deflection. This leads to a change of the robot pose and the robot paths during operation, which is detrimental to accuracy. The overall impact of the heat on the robot structure was recorded by measuring the position of the TCP during measurement motions of the flying cutting device. Both the relative TCP 
displacement in $\mathrm{x}, \mathrm{y}$ and $\mathrm{z}$ direction and the relative total error as compared to the initial measurement are shown in Figure 9 on the basis of measurements at two different measuring points.

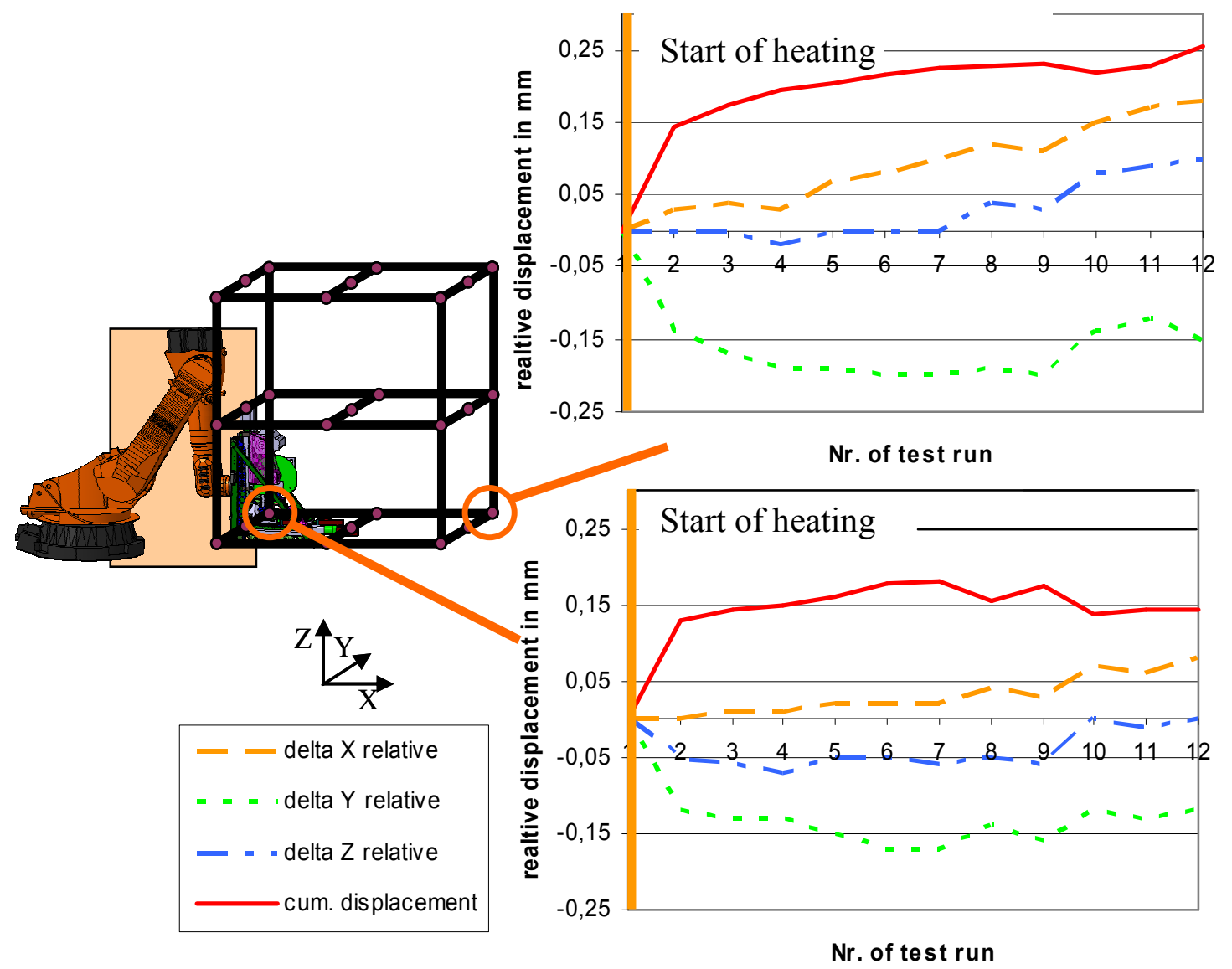

Figure 9: TCP displacement, partial heating (wbk)

Figure 9 shows an increase in TCP deviation during the first two measurements. As displayed in Figures 7 and 8, this is caused by the stronger temperature increase of the robot structure during the heat-up phase. Afterwards, the temperature increase decelerates and the TCP deviation rises to a smaller extent. The total additional displacement of the TCP at the examined measuring points caused by the temperature-induced deformation affecting the robot amounts up to $0.25 \mathrm{~mm}$.

The TCP displacement in y direction is more pronounced than in $\mathrm{x}$ and $\mathrm{z}$. The development of TCP displacement in $\mathrm{x}$ and $\mathrm{z}$ direction is mainly caused by the change in length of both the lower and the upper robot arm. In Figure 10, this development has been tested and proved by setting up a model. The robot structure was adjusted according to the first measuring point by means of a simplified model consisting of two thermally variable bars corresponding to the robot arms in terms of length. The elongation of the bars caused by an averaged temperature increase from the measured values at the front and back side of the robot were used to calculate the respective displacement of the TCP. 


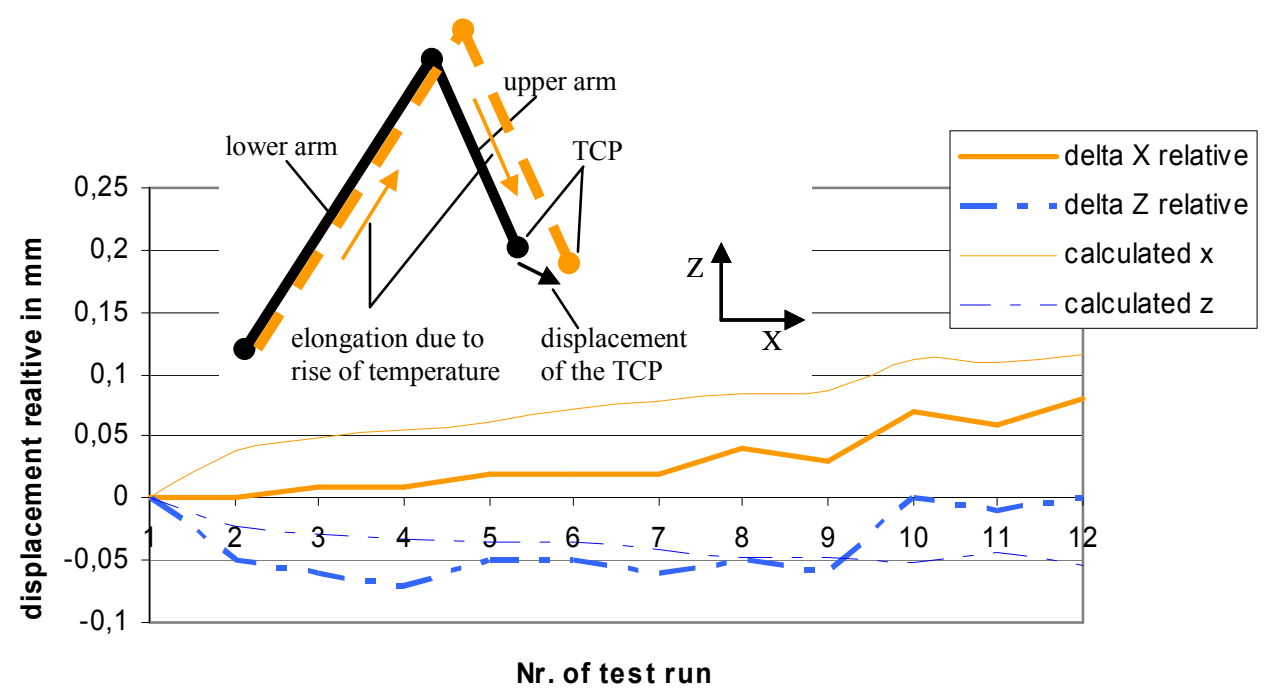

Figure 10: Comparison of calculated and measured TCP displacement in y and z direction (wbk)

The calculation from the simplified model in Figure 10 shows a good agreement between measured and calculated values in $\mathrm{x}$ and $\mathrm{y}$ direction. The discrepancy with the measured values can be explained by the model with some simplification, as not all the components of the flying cutting device were taken into consideration. Figure 9 shows that TCP displacement is mainly caused, though, by the large displacement of the y position in the negative direction. The deviations in this direction may amount up to $0.2 \mathrm{~mm}$. This displacement cannot be calculated by means of the simplified model, because the temperature difference between front and back side leads to the thermal deflection of the heated components.

When evaluating the measurement results, it should be considered that the intrinsic heating of the robot drives needs to be added to the identified thermal deformations during real operation. Besides, prior to measurements, the position of the robot was chosen to allow the thermal changes in length of the oscillations to compensate each other to the largest possible extend due to their spatial orientation. This effect can be seen in Figure 10.

Previous measurements show that the heating of the robot axes causes an additional displacement of the TCP in the robot poses. The deformations of the robot axes caused by heating affect the path of the flying cutting device during movement. As mentioned above, however, the final contour of the profile is directly dependent on the path taken by the flying cutting device during CPE regarding the production of extrusded profiles. The mere numerical calibration of the motion paths of the flying cutting device and, thus, error compensation based on the absolute accuracy of the robot would not be sufficient to minimize errors during the production of extruded profiles and increase their accuracy. The measurements carried out up to date show further potential through an additional compensation of the errors caused by temperature increases, whereas it needs to be taken into account that a good positioning of the robot may already reduce the thermal deformations considerably. Following work will try to achieve the mathematical description of the overall structure in order to calculate the influences of heating resulting from both process and drives on thermal deflection. One approach to the accuracy increase of robot paths and extruded profiles will then consist in the use of the conceived mathematical model for path generation through the consideration of temperature increases at the flying cutting device and the robot.

An integrated calibration approach for the improvement of the accuracy allows for the temperature-related influences to be additionally accounted for by means of a supplementary learning in-line calibration during the production process. Previously developed and established methods for the inline calibration of robot applications cannot be applied to the flying cutting device used for the extrusion process. These methods are mostly based on a calibration system working with a reference object that is both temperature stable and distortion-free. During working mode of the robot cell, additional sensors fitted to the robot measure the robot-pose which is to be calibrated via previously 
determined poses in relation to the reference object. Subsequently, the sensors identify a possible deviation of the actual pose from the reference pose. The robot model stored in the control is corrected to match the measured sensor values on the basis of a suitable algorithm. In the case of extrusion, the flying cutting device is required to continuously support the manufactured profile, which impedes any movement towards a reference object during operation. A calibration strategy like this cannot be used for this application.

One approach to a learning in-line calibration of the flying cutting device would consist in the use of measurement data from the downstream quality assurance of the extruded profiles. Deviations of the extruded profile would be recorded right after cutting and transfer and then stored in an error model on the basis of a suitable method linking the error to the movement and position of the robot at a specific time. As mentioned above, accuracy requirements are not equally high in different locations of the working envelope. In accordance with the law of the lever, the accuracy needs to be higher close to the guiding tool of the extrusion press than at a greater distance from it. Statistically, calibration accuracy is rising close to the guiding tool and falling when moving away from it because of the higher probability of the robot acting in the front section of the working envelope close to the extrusion press. An iterative method allows for a gradual increase of the absolute accuracy with every extruded and measured profile and for the continuous compensation of thermal modifications in particular.

\section{Summary and outlook}

This article presents the possibility of manufacturing curved aluminum extruded profiles by using a flying cutting device and a kinematic transfer mechanism. Contour deviations shown by the profiles that have been manufactured so far facilitated the determination and examination of factors affecting contour accuracy. The accuracies and errors in position deviations achieved up to date were determined by means of the direct impact of a position deviation of the flying cutting device on the target profile contour and measurements. The necessity of calibration was identified and explained on the basis of these measurements regarding the statistical accuracies of the robots operating in the working envelope in front of the extrusion press and of examinations of the effects of partial heating resulting from the extrusion press that affects robot accuracy. On the basis of these measurement results, approaches to static calibration and to a learning in-line calibration were presented.

Future work will be dedicated to investigations how the measurement results can be used for the calibration of robot paths with the aim to increase profile accuracy in a first step through improving the accuracy in the reference paths of the flying cutting device. Besides this, the details of the approach to a learning in-line calibration and to a mathematical description of the flying cutting device are to be worked out in order to be eventually able to compensate temperature-related influences on kinematic structures during operation.

Furthermore, already initiated tests on dynamic deviations during profile manufacturing have to be extended by the synchronous recording of robot paths during the manufacturing of extruded profiles. Subsequently, the results create the basis for accuracy improvements of the overall system by extending existing models.

\section{Acknowledgement}

This paper is based on investigations of the Collaborative Research Center SFB/TR10 which is kindly supported by the German Research Foundation (DFG). 


\section{References}

[1] Kleiner, M; Klaus, A.; Becker, D.: Innovative Fertigung von 3D-gekrümmten Strangpressprofilen. ZWF 98 (2003) H. 10, S. 476ff.

[2] Fleischer, J.; Munzinger, C.; Schneider, M.; Stengel, G.: Fliegendes Abtrennen, In: Fortschrittsberichte VDI Reihe 2 Nr. 661, VDI-Verlag Düsseldorf, 2007, ISBN 9783183661022

[3] Fleischer, J.; Schmidt-Ewig, J. P.: Innovative Machine Kinematics for Combined Handling and Machining of Three-Dimensional Curved Lightweight Extrusion Structures, Annals of the CIRP 54 (2005), pp. 317-320

[4] Fleischer, J.; Munzinger, C.; Stengel, G.: Flying Cutting of Spatially Curved Extrusion Profiles, In: Advanced Materials Research: Flexible Manufacture of Lightweight Frame Structures, Band 10 (2006), pp. 35-42

[5] Klaus. A.; Becker, D.; Kleiner, M.: Three-Dimensional Curved Profile Extrusion - First Results on the Influence of Gravity In: Advanced Materials Research: Flexible Manufacture of Lightweight Frame Structures, Band 10 (2006), pp. 5-12

[6] BMBF-Projekt 02PP2410: „Gerundete Strangpressprofile aus Al- und Mg-Legierungen für ultraleichte Tragwerke, BMBF Abschlussbericht, 2005

[7] Becker, D.; Kleiner, M.; Schikorra, M.: Mehrachsiges Runden beim Strangpressen In: Fortschrittsberichte VDI Reihe 2 Nr. 661, VDI-Verlag Düsseldorf, 2007, ISBN 9783183661022

[8] DIN EN ISO 9283: Industrieroboter - Leistungskenngrößen und zugehörige Prüfmethoden, 1998

[9] Wiest, U.: Kinematische Kalibrierung von Industrierobotern, Dissertation, Shaker-Verlag Aachen, 2001. ISBN 3826586093

[10] Bongardt, T.: Methode zur Kompensation betriebsabhängiger Einflüsse auf die Absolutgenauigkeit von Industrierobotern, Dissertation, Herbert Utz Verlag, 2003, ISBN 3831603324

[11] Dauster, Katja :Prozessangepasste, lernende Roboterregelung für Montageprozesse, In: Fortschrittsberichte VDI Nr. 925, VDI-Verlag Düsseldorf, 2002, ISBN 3183925087 